\title{
Etiologia e quantificação dos agentes causais de manchas foliares na cultura do trigo nas safras 2008 a 2011
}

\author{
Rosane Fátima Baldiga Tonin ${ }^{1}$; Erlei Melo Reis ${ }^{1,2}$; Anderson Luiz Durante Danelli ${ }^{1}$
}

${ }^{1}$ Faculdade de Agronomia e Medicina Veterinária, Laboratório de Fitopatologia - Micologia, Universidade de Passo Fundo - UPF, 99001, Passo Fundo, RS, Brasil. ${ }^{2}$ Professor da FAMV/PPGAgro/UPF.

Autor para correspondência: Rosane Fátima Baldiga Tonin (rosanetonin@yahoo.com.br)

Data de chegada: 09/01/2013. Aceito para publicação em: 13/04/2013.

1865

\section{RESUMO}

Tonin, R.F.B.; Reis, E.M.; Danelli, A.L.D. Etiologia e quantificação dos agentes causais de manchas foliares na cultura do trigo nas safras 2008 a 2011. Summa Phytopathologica, v.39, n.2, p.102-109, 2013.

A cultura do trigo é uma das opções mais importantes para cultivo na safra de inverno. Entre as doenças foliares a mancha-amarela da folha, a mancha marrom e a septoriose são citadas como as mais frequentes em trigo. Este trabalho teve como objetivo identificar e quantificar os fungos fitopatogênicos associados a sintomas de manchas foliares em cultivares de trigo, nas Regiões tritícolas de Valor de Cultivo e Uso (VCU). Foram analisadas 162 amostras coletadas nas safras 2008 a 2011, oriundas dos Estados do Paraná, Santa Catarina, Minas Gerais, São Paulo e Rio Grande do Sul. Discos foliares assépticos, 25 por amostra, foram distribuídos em gerbox, constituindo uma câmara úmida e incubados a temperatura de $25^{\circ} \mathrm{C}$ e fotoperíodo de 12 horas. Após um período de incubação de oito dias, foi realizada a avaliação, identificando e quantificando a incidência dos fungos presentes nos discos foliares. Constatou-se a ocorrência de Bipolaris sorokiniana, Drechslera triticirepentis, D. siccans e Stagonospora nodorum associados às lesões foliares em trigo. Verificou-se na safra 2008, a predominância de D. siccans com incidência de 0 a $75 \%$ nas amostras avaliadas, sendo o primeiro relato desta espécie, em trigo, no Brasil. Na média das safras avaliadas $B$. sorokiniana apresentou incidência de $7,6 \%$ e frequência de $53,1 \%, D$. tritici-repentis apresentou incidência de 59,2\% e frequência de 90,6\%, D. siccans incidência de $11,0 \%$ e freqüência de $48,1 \%$ e $S$. nodorum com incidência e frequência de $1,55 \%$ e de $2,2 \%$, respectivamente.

Palavras-chave adicionais: Triticum aestivum, Drechslera, Bipolaris, Stagonospora, ocorrência.

\section{ABSTRACT}

Tonin, R.F.B.; Reis, E.M.; Danelli, A.L.D. Etiology and quantification of causal agents of wheat leaf spots in 2008 to 2011 growing seasons. Summa Phytopathologica, v.39, n.2, p.102-109, 2013.

The wheat (Triticum aestivum L.) crop is one of the most important options for cultivation in the winter season. Among the foliar diseases, yellow leaf spot, brown-spot and septoriosis, are cited as the most frequent leaf spot in wheat. This study aimed to identify and quantify the pathogenic fungi associated with leaf spot symptoms in wheat cultivars in Regions of Value for Cultivation and Use (RVCU). One hundred and sixty two samples collected in 2008 to 2011 season, in wheat field in the states of Paraná, Santa Catarina, Minas Gerais, São Paulo and Rio Grande do Sul were analysed. Twenty five aseptic leaf discs per sample were distributed in a moist chamber within a gerbox and incubated at $25^{\circ} \mathrm{C}$ and 12 hours photoperiod. After incubation for eight days the identification and quantification of the fungi incidence present in leaf discs were performed. In the 2008 season, there was a predominance of Drechslera siccans with an incidence of 0 to $75 \%$, being the first report of this species in wheat, Brazil. On the seasons average B. sorokiniana showed a $7.6 \%$ incidence and $53.1 \%$ frequency, D. tritici-repentis presented an incidence of $59.2 \%$ and $90.6 \%$ frequency, D. siccans incidence of $11.0 \%$ and $48.1 \%$ frequency, and $S$. nodorum with incidence and frequency of $1.55 \%$ and $2.2 \%$, respectively.

Additional keywords: Triticum aestivum, Drechslera, Bipolaris, Stagonospora, occurrence.

O trigo (Triticum aestivum L.) ocupa papel de destaque dentre os cereais produzidos no Brasil, tendo uma importante função econômica e social. Apesar dessa importância, seu potencial produtivo não tem sido explorado devidamente (1).

A produção nacional de trigo na safra $2011 / 12$ foi de 5.788 milhões de toneladas, com rendimento médio de $2.672 \mathrm{Kg} / \mathrm{ha}$ e o consumo de 11 milhões de toneladas. A área cultivada foi de 2.166,2 mil hectares, distribuídos nas regiões centro-oeste, sul e sudeste (5).

As limitações ocorrentes para produzir trigo são a suscetibilidade dos cultivares, a agressividade dos fungos agentes causais de doenças, o excesso de chuva, o acúmulo de dias nublados e a monocultura em plantio direto favoráveis à ocorrência e ao aumento da intensidade das doenças (11).

Devido às condições ambientais adversas, aliadas à suscetibilidade das cultivares e dependendo das práticas culturais, o trigo pode ter seu rendimento prejudicado pelo ataque de doenças causadas por fungos. Os danos no rendimento e na qualidade dos grãos são maiores quando várias doenças ocorrem simultaneamente na lavoura. Entre estas, destacam-se as que incidem na parte aérea da cultura, como a ferrugem, o oídio e as manchas foliares (13).

Os danos causados pelas doenças foliares são atribuídos principalmente à redução da atividade fotossintética da planta (2). O 
oídio [Blumeria (Sin. Erysiphe) graminis Speer f.sp. tritici Marchal], a ferrugem da folha (Puccinia triticina Eriks.), a mancha-amarela [Drechslera tritici-repentis (Died.) Drechs.], a mancha marrom [Bipolaris sorokiniana (Sacc.) Shoem.] e a septoriose [Stagonospora nodorum (Berk.) Berk.], são as principais doenças foliares na Região Sul do Brasil (14).

As principais manchas foliares são a mancha-amarela, a manchamarrom e a septoriose. Os agentes causais causam lesões necróticas com halo clorótico nas folhas e sobrevivem em semente e restos culturais. As manchas foliares ocorrem e têm maior intensidade quando são utilizadas sementes infectadas ou quando o trigo é cultivado sob monocultura em sistema de plantio direto $(12 ; 17)$.

A redução na produtividade pode atingir até $80 \%$ para mancha marrom (8), até $48 \%$ para mancha amarela (10) e até $31 \%$ para septoriose (3).

O presente trabalho teve como objetivo identificar e quantificar os fungos fitopatogênicos associados a sintomas de manchas foliares em cultivares de trigo, nas regiões de Valor de Cultivo e Uso (VCU), I, II, III e IV, no período de 2008 a 2011.

\section{MATERIAL E MÉTODOS}

O trabalho foi conduzido no Laboratório de Fitopatologia Micologia da Faculdade de Agronomia e Medicina Veterinária da Universidade de Passo Fundo - RS, nos anos de 2008 a 2011.

Amostras de folhas de trigo com sintomas de manchas foliares, provenientes de lavouras de trigo do estado do Paraná, Santa Catarina, Minas Gerais, São Paulo e Rio Grande do Sul, foram analisadas nos anos de 2008, 2009, 2010 e 2011. Daquelas, 16 amostras foram da safra de 2008, 76 de 2009, 36 de 2010 e 34 de 2011, totalizando 162 amostras.

Para o isolamento foram cortados discos foliares contendo parte da mancha e do tecido sadio, de 9,0 mm de diâmetro e submetidos à assepsia. A assepsia dos discos foliares foi realizada pela imersão em solução de álcool 99\%, lavando-os ligeiramente em água destilada e, em seguida, transferidos para uma solução aquosa de hipoclorito de sódio $1 \%$, por três minutos e, novamente lavados com água esterilizada. Posteriormente, 25 discos/amostra foram distribuídos em caixas de acrílico, tipo gerbox de poliestireno cristal $(11 \times 11 \mathrm{x}$ $3,5 \mathrm{~cm}$ de altura), contendo no fundo do recipiente uma camada de espuma de nylon $(5 \mathrm{~mm}$ ) e duas folhas sobrepostas de papel filtro, saturadas com água destilada e esterilizada, constituindo uma câmara úmida.

A incubação foi conduzida em ambiente controlado, câmara de crescimento com temperatura de $25^{\circ} \mathrm{C}$ e fotoperíodo de 12 horas proporcionadas por três lâmpadas fluorescentes OSRAM Universal, 40 watts de potência, localizadas a $50 \mathrm{~cm}$ acima dos gerbox.

Após um período de incubação de oito dias, foi realizada a avaliação, com um microscópio estereoscópico, identificando e quantificando a incidência dos fungos presentes nos discos foliares. Por incidência entende-se o número de discos que estavam doentes, expresso em percentagem ou proporção, do número total de unidades avaliadas (4).

\section{RESULTADOS E DISCUSSÃO}

Associados ao quadro sintomático de folhas de trigo foram identificados quatro fungos agentes causais de manchas foliares: $B$. sorokiniana, D. tritici-repentis, D. siccans e $S$. nodorum.

Analisando as amostras referentes à safra agrícola de 2008 (Tabela 1), B. sorokiniana apresentou incidência de 0 a $30 \%$ (média de 5,9\%), D. tritici-repentis de 0 a $72 \%$ (média de $18,0 \%$ ) e D. siccans de 0 a 75 $\%$ (média de $36,0 \%$ ).

Tabela 1. Incidência ${ }^{\mathrm{x}}$ de fungos associados a manchas foliares em cultivares de trigo na região de VCU I, safra agrícola 2008.

\begin{tabular}{|c|c|c|c|c|c|}
\hline Cultivar & Local & $\begin{array}{l}\text { Região } \\
(\mathrm{VCU})^{\mathrm{y}}\end{array}$ & $\begin{array}{c}\text { Bipolaris } \\
\text { sorokiniana }\end{array}$ & $\begin{array}{c}\text { Drechslera } \\
\text { tritici-repentis }\end{array}$ & $\begin{array}{c}\text { Drechslera } \\
\text { siccans }\end{array}$ \\
\hline Pampeano & Coxilha/RS & $\mathrm{I}$ & 0 & 0 & 75 \\
\hline Fund $.30^{z}$ & L. Vermelha/RS ${ }^{z}$ & I & 0 & 0 & 30 \\
\hline Quartzo & M. Castelhano/ RS ${ }^{z}$ & I & 0 & 0 & 52 \\
\hline Quartzo & Coxilha/RS & I & 0 & 48 & 4 \\
\hline Abalone & Vacaria/RS & I & 0 & 4 & 24 \\
\hline Supera & L. Vermelha/RS & I & 4 & 0 & 50 \\
\hline Raízes & L. Vermelha/RS & I & 0 & 0 & 50 \\
\hline Louro & L. Vermelha/RS & I & 12 & 4 & 60 \\
\hline Abalone & Passo Fundo/RS & I & 8 & 65 & 16 \\
\hline Marfim & Vacaria/RS & I & 8 & 0 & 72 \\
\hline Supera & Vacaria/RS & I & 0 & 4 & 60 \\
\hline Guamirim & L. Vermelha/RS & I & 16 & 4 & 75 \\
\hline Safira & P. das Missões/RS ${ }^{z}$ & I & 4 & 12 & 0 \\
\hline Guabijú & L. Vermelha/RS & I & 10 & 25 & 4 \\
\hline Fund $.52^{2}$ & Coxilha/RS & I & 30 & 50 & 4 \\
\hline Safira & Passo Fundo/RS & I & 3 & 72 & 0 \\
\hline Média & & & 5,9 & 18,0 & 36,0 \\
\hline Frequência & & & 56,2 & 62,5 & 87,5 \\
\hline
\end{tabular}

${ }^{\mathrm{x}}$ Incidência (\%) determinada em discos de 9,0 mm de diâmetro recortados de manchas foliares e incubados 25 por amostra. ${ }^{\mathrm{y}}$ Valor de Cultivo e Uso. ${ }^{\text {}}$ Fundacep 30, Fundacep 52, Lagoa Vermelha, Mato Castelhano, Palmeira das Missões. 
Nas amostras analisadas no ano de 2009 (Tabela 2) foi observado B. sorokiniana com incidência de 0 a $100 \%$ (média de $15,0 \%$ ), $D$. tritici-repentis com incidência de 12 a $100 \%$ (média de $66,1 \%$ ), e $D$. siccans de 0 a $24 \%$ (média de $3,1 \%$ ).

$\mathrm{Na}$ safra de 2010, a incidência de $B$. sorokiniana oscilou entre 0 a $21 \%$ (média de 2,2\%), D. tritici-repentis entre 0 a $100 \%$ (média de
$86,4 \%$ ) e $D$. siccans entre 0 a $16 \%$ (média de $2,1 \%$ ) (Tabela 3 ).

$\mathrm{Na}$ avaliação das amostras do ano 2011, foi observada incidência de $B$. sorokiniana de 0 a $35 \%$ (média de $7,5 \%$ ), de 0 a $96 \%$ (média de $66,3 \%$ ) para $D$. tritici-repentis, de 0 a $20 \%$ (média de $3,1 \%$ ), para $D$. siccans e de 0 a $100 \%$ (média de 6,2\%) e para S. nodorum (Tabela 4).

Quando se considera as regiões tritícolas de VCU (6), foram

Tabela 2. Incidência ${ }^{\mathrm{x}}$ de fungos associados a manchas foliares em cultivares de trigo nas regiões VCU I, II, III, na safra agrícola 2009.

\begin{tabular}{|c|c|c|c|c|c|}
\hline Cultivar & Local & $\begin{array}{l}\text { Região } \\
(\text { VCU })^{y}\end{array}$ & $\begin{array}{c}\text { Bipolaris } \\
\text { sorokiniana }\end{array}$ & $\begin{array}{c}\text { Drechslera } \\
\text { tritici-repentis }\end{array}$ & $\begin{array}{c}\text { Drechslera } \\
\text { siccans }\end{array}$ \\
\hline Safira & Coxilha/RS & I & 12 & 72 & 24 \\
\hline Ônix & Coxilha/RS & I & 32 & 40 & 8 \\
\hline Abalone & Coxilha/RS & I & 20 & 52 & 12 \\
\hline Fundacep 52 & Espumoso/RS & I & 0 & 67 & 0 \\
\hline Safira & Coxilha/RS & I & 4 & 44 & 8 \\
\hline Mirante & Coxilha/RS & I & 8 & 40 & 4 \\
\hline Marfim & Coxilha/RS & I & 12 & 40 & 4 \\
\hline BRS 296 & Carazinho/RS & I & 12 & 75 & 0 \\
\hline Quartzo & Coxilha/RS & I & 20 & 12 & 0 \\
\hline Quartzo & Carazinho/RS & I & 12 & 81 & 0 \\
\hline Ônix & Ciríaco/RS & I & 20 & 86 & 12 \\
\hline Valente & Coxilha/RS & I & 0 & 32 & 0 \\
\hline Guamirim & Carazinho/RS & I & 12 & 24 & 0 \\
\hline Nova Era & Ibiraiaras/RS & I & 4 & 80 & 16 \\
\hline Marfim & Carazinho/RS & I & 16 & 60 & 12 \\
\hline Quartzo & Chapada/RS & I & 40 & 52 & 4 \\
\hline Abalone & Chapada/RS & I & 20 & 100 & 0 \\
\hline Pampeano & Coxilha/RS & I & 16 & 38 & 16 \\
\hline Marfim & Chapada/RS & I & 16 & 96 & 4 \\
\hline Campeiro & Chapada/RS & I & 12 & 94 & 8 \\
\hline Quartzo & Carazinho/RS & I & 20 & 20 & 12 \\
\hline Raízes & Carazinho/RS & I & 4 & 24 & 0 \\
\hline Mirante & Chapada/RS & I & 24 & 96 & 0 \\
\hline Campeiro & Ciríaco/RS & I & 0 & 12 & 0 \\
\hline Quartzo & Ciríaco/RS & I & 60 & 24 & 12 \\
\hline Mirante & Ciríaco/RS & I & 92 & 28 & 0 \\
\hline Safira & Ciríaco/RS & I & 8 & 32 & 12 \\
\hline Abalone & P. Fundo/RS ${ }^{z}$ & I & 4 & 84 & 4 \\
\hline Ônix & Ciríaco/RS & I & 24 & 44 & 12 \\
\hline Guamirim & Tucunduva/RS & II & 4 & 92 & 0 \\
\hline Raízes & Tucunduva/RS & II & 100 & 20 & 0 \\
\hline Fundacep 52 & Tucunduva/RS & II & 76 & 96 & 4 \\
\hline Mirante & Tucunduva/RS & II & 8 & 96 & 0 \\
\hline Quartzo & Tucunduva/RS & II & 8 & 85 & 0 \\
\hline Safira & S. Rosa/RS ${ }^{z}$ & II & 0 & 100 & 0 \\
\hline BRS 296 & C. Novo/RS & II & 95 & 40 & 0 \\
\hline Cristalino & Tucunduva/RS & II & 4 & 100 & 0 \\
\hline Guamirim & C. Novo/RS & II & 14 & 96 & 8 \\
\hline BRS 208 & C. Novo/RS & II & 4 & 92 & 8 \\
\hline Safira & C. Novo/RS & II & 0 & 100 & 0 \\
\hline Horizonte & Tucunduva/RS & II & 46 & 100 & 0 \\
\hline
\end{tabular}


Tabela 2. Incidência ${ }^{\mathrm{x}}$ de fungos associados a manchas foliares em cultivares de trigo nas regiões VCU I, II, III, na safra agrícola 2009.

\begin{tabular}{|c|c|c|c|c|c|}
\hline Cultivar & Local & $\begin{array}{l}\text { Região } \\
(\text { VCU })^{y}\end{array}$ & $\begin{array}{c}\text { Bipolaris } \\
\text { sorokiniana }\end{array}$ & $\begin{array}{c}\text { Drechslera } \\
\text { tritici-repentis }\end{array}$ & $\begin{array}{c}\text { Drechslera } \\
\text { siccans }\end{array}$ \\
\hline CD 117 & C. Novo/RS & II & 16 & 76 & 4 \\
\hline CD 114 & C. Novo/RS & II & 12 & 100 & 12 \\
\hline Cristalino & C. Novo/RS & II & 12 & 80 & 0 \\
\hline Raízes & Ijuí/RS & II & 8 & 96 & 4 \\
\hline Horizonte & C. Novo/RS & II & 4 & 100 & 8 \\
\hline Raízes & C. Novo/RS & II & 8 & 96 & 0 \\
\hline Quartzo & Ijuí/RS & II & 4 & 100 & 0 \\
\hline Marfim & Ijuí/RS & II & 0 & 100 & 0 \\
\hline 7051 & Faxinal/PR & II & 0 & 100 & 0 \\
\hline Asteca & Faxinal/PR & II & 0 & 72 & 0 \\
\hline 6022 & Faxinal/PR & II & 8 & 68 & 0 \\
\hline CD 104 & Faxinal/PR & II & 12 & 52 & 0 \\
\hline Bio 06007 & Faxinal/PR & II & 0 & 68 & 0 \\
\hline BRS 208 & Faxinal/PR & II & 8 & 52 & 0 \\
\hline BRS 220 & Faxinal/PR & II & 28 & 40 & 0 \\
\hline Valente & Faxinal/PR & II & 4 & 52 & 0 \\
\hline 6027 & Faxinal/PR & II & 0 & 76 & 0 \\
\hline 7218 & Faxinal/PR & II & 0 & 80 & 0 \\
\hline 6016 & Faxinal/PR & II & 4 & 84 & 0 \\
\hline 7052 & Faxinal/PR & II & 0 & 80 & 0 \\
\hline 6011 & Faxinal/PR & II & 0 & 80 & 0 \\
\hline 6004 & Faxinal/PR & II & 0 & 85 & 0 \\
\hline 6014 & Faxinal/PR & II & 0 & 80 & 0 \\
\hline Quartzo & Faxinal/PR & II & 0 & 89 & 0 \\
\hline Mirante & Faxinal/PR & II & 0 & 100 & 0 \\
\hline Mirante & Ventania/PR & II & 0 & 12 & 0 \\
\hline Ônix & Ventania/PR & II & 0 & 60 & 0 \\
\hline Asteca & Cambé/PR & III & 0 & 48 & 0 \\
\hline Mirante & Cambé/PR & III & 0 & 56 & 0 \\
\hline IPR 136 & Cambé/PR & III & 4 & 48 & 0 \\
\hline Supera & Cambé/PR & III & 0 & 16 & 0 \\
\hline Valente & Cambé/PR & III & 0 & 24 & 0 \\
\hline Quartzo & Cambé/PR & III & 4 & 36 & 0 \\
\hline BRS Tangará & Apucarana/PR & III & 54 & 68 & 0 \\
\hline CD 116 & Apucarana/PR & III & 61 & 86 & 0 \\
\hline Média & & & 15,0 & 66,1 & 3,1 \\
\hline Frequência & & & 69,7 & 100,0 & 32,9 \\
\hline
\end{tabular}

Incidência (\%) determinada em discos de 9,0 mm de diâmetro recortados de manchas foliares e incubados 25 por amostra. ${ }^{\mathrm{y}}$ Valor de Cultivo e Uso. ${ }^{\mathrm{z}}$ Campo Novo, Passo Fundo, Santa Rosa.

constatado na safra 2008 , frequências de $B$. sorokiniana de $56,2 \%, D$. tritici-repentis de $62,5 \%$ e D. siccans de $87,5 \%$ (Tabela 1 ), na região VCU I.

Considerando a safra de 2009 , a frequência de $B$. sorokiniana foi de $69,7 \%$, D. tritici-repentis de $100,0 \%$ e D. siccans de $32,9 \%$ (Tabela 2), na região de VCU I, II e III. Na safra 2010 a frequência de B. sorokiniana foi de $30,5 \%$, D. tritici-repentis de $100,0 \%$ e $D$. siccans de 33,3 \% (Tabela 3), na região de VCU I, II e III e frequência de $55,9 \%$ de $B$. sorokiniana, $100,0 \%$ de $D$. tritici-repentis, $38,2 \%$ de D. siccans e $S$. nodorum de $8,8 \%$, respectivamente, na safra 2011(Tabela 4) na região de VCU I, II, III e IV.

Observou-se a ocorrência dos fungos $B$. sorokiniana e D. triticirepentis nas regiões de VCU I, considerada fria/úmida/alta, região de VCU II sendo moderadamente quente/úmida/baixa, região de VCU III, considerada quente/moderadamente seca/baixa do estado e região de VCU IV sendo quente/seca - Cerrado. Drechslera siccans foi detectado 
Tabela 3. Incidência ${ }^{\mathrm{x}}$ de fungos associados a manchas foliares em cultivares de trigo nas regiões VCU I, II, III, na safra agrícola 2010.

\begin{tabular}{|c|c|c|c|c|c|}
\hline Cultivar & Local & $\begin{array}{l}\text { Região } \\
(\operatorname{VCU})^{y}\end{array}$ & $\begin{array}{c}\text { Bipolaris } \\
\text { sorokiniana }\end{array}$ & $\begin{array}{c}\text { Drechslera } \\
\text { tritici-repentis }\end{array}$ & $\begin{array}{c}\text { Drechslera } \\
\text { siccans }\end{array}$ \\
\hline Horizonte & J.Castilhos/RS ${ }^{\text {Z }}$ & I & 0 & 96 & 12 \\
\hline Safira & Passo Fundo/RS & I & 0 & 93 & 3 \\
\hline Safira & Coxilha/RS & I & 0 & 100 & 4 \\
\hline Safira & Vacaria/RS & I & 0 & 100 & 4 \\
\hline Mirante & Vacaria/RS & I & 0 & 74 & 0 \\
\hline Fundacep 52 & Vacaria/RS & I & 0 & 92 & 4 \\
\hline Pioneiro & Passo Fundo/RS & I & 18 & 75 & 4 \\
\hline Guamirim & J. Castilhos/RS & I & 4 & 42 & 16 \\
\hline Mirante & Passo Fundo/RS & I & 0 & 93 & 0 \\
\hline BRS 220 & C. Mourão/PR ${ }^{z}$ & II & 0 & 88 & 0 \\
\hline Quartzo & C. Mourão/PR & II & 4 & 96 & 0 \\
\hline Vaqueano & S. Augusto/RS ${ }^{z}$ & II & 0 & 94 & 5 \\
\hline Itaipú & S. Augusto/RS & II & 0 & 86 & 0 \\
\hline Alcover & S. Augusto/RS & II & 0 & 84 & 0 \\
\hline Raízes & Panambi/RS & II & 21 & 67 & 12 \\
\hline Ivaí & S. Augusto/RS & II & 0 & 85 & 0 \\
\hline Abalone & S. Augusto/RS & II & 0 & 85 & 7 \\
\hline Abalone & Ventania/PR & II & 0 & 64 & 3 \\
\hline Quartzo & Ventania/PR & II & 0 & 79 & 0 \\
\hline Mirante & Ventania/PR & II & 4 & 83 & 0 \\
\hline Mirante & S. Augusto/RS & II & 0 & 83 & 0 \\
\hline Ônix & S. Augusto/RS & II & 0 & 92 & 0 \\
\hline Safira & Entre Ijuís/RS & II & 4 & 83 & 3 \\
\hline Quartzo & Faxinal/PR & II & 0 & 40 & 0 \\
\hline Safira & Piratuba/SC & II & 4 & 100 & 0 \\
\hline Valente & Pitangueiras/PR & III & 4 & 100 & 0 \\
\hline Taurum & Pitangueiras/PR & III & 8 & 100 & 0 \\
\hline CD 104 & Pitangueiras/PR & III & 4 & 100 & 0 \\
\hline Bio 007051 & Pitangueiras/PR & III & 4 & 100 & 0 \\
\hline Abalone & Pitangueiras/PR & III & 0 & 86 & 0 \\
\hline Ivaí & Pitangueiras/PR & III & 0 & 85 & 0 \\
\hline Itaupú & Pitangueiras/PR & III & 0 & 79 & 0 \\
\hline Mirante & Pitangueiras/PR & III & 0 & 92 & 0 \\
\hline BRS Tangará & Pitangueiras/PR & III & 0 & 100 & 0 \\
\hline BRS Pardela & Pitangueiras/PR & III & 0 & 100 & 0 \\
\hline BRS 208 & Pitangueiras/PR & III & 0 & 96 & 0 \\
\hline Média & & & 2,2 & 86,4 & 2,1 \\
\hline Frequência & & & 30,5 & 100,0 & 33,3 \\
\hline
\end{tabular}

${ }^{x}$ Incidência (\%) determinada em discos de $9,0 \mathrm{~mm}$ de diâmetro recortados de manchas foliares e incubados 25 por amostra. ${ }^{y}$ Valor de Cultivo e Uso. ${ }^{\mathrm{C}}$ Campo Mourão, Júlio de Castilhos, Santo Augusto.

nas regiões VCU I e II, não sendo encontrado na região VCU III e IV, sendo o mesmo observado para $S$. nodorum.

O fungo $D$. siccans foi constatado pela primeira vez em amostras foliares de trigo, constituindo assim, o primeiro relato da ocorrência dessa espécie no Brasil. Anteriormente, este gênero era encontrado somente em azevém (Lolium multiflorum L.) e, atualmente, está causando danos na cultura do trigo. Nas amostras do ano de 2008 a incidência e a frequência de $D$. siccans foram superiores a $D$. tritici- repentis e a $B$. sorokiniana (Tabela 1$)$.

Stolte (16), em trabalho de determinação da etiologia de manchas foliares do trigo na safra de 2005 , detectou a presença de uma outra espécie de Drechslera distinta de $D$. tritici-repentis, provavelmente D. siccans, com incidência de 2,0 a $20,0 \%$.

Amostras correspondentes à safra agrícola 2009 apresentaram incidência e frequência superiores de $D$. tritici-repentis em relação à $B$. sorokiniana e à $D$. siccans (Tabela 2 ). 
Tabela 4. Incidência ${ }^{\mathrm{x}}$ de fungos associados a manchas foliares em cultivares de trigo nas regiões VCU I, II, III e IV na safra agrícola 2011.

\begin{tabular}{|c|c|c|c|c|c|c|}
\hline Cultivar & Local & $\begin{array}{l}\text { Região } \\
(\mathrm{VCU})^{y}\end{array}$ & $\begin{array}{c}\text { Bipolaris } \\
\text { sorokiniana }\end{array}$ & $\begin{array}{c}\text { Drechslera } \\
\text { tritici-repentis }\end{array}$ & $\begin{array}{l}\text { Drechslera } \\
\text { siccans }\end{array}$ & $\begin{array}{l}\text { Stagonospora } \\
\text { nodorum }\end{array}$ \\
\hline Mirante & M.Castelhano/RS & I & 0 & 88 & 0 & 12 \\
\hline Safira & Erebango/RS & I & 0 & 89 & 3 & 0 \\
\hline Marfim & Passo Fundo/RS & I & 26 & 65 & 0 & 0 \\
\hline Mirante & Passo Fundo/RS & I & 9 & 82 & 3 & 0 \\
\hline Safira & M.Castelhano/RS & I & 0 & 94 & 0 & 0 \\
\hline Marfim & P. Missões/RS ${ }^{z}$ & I & 0 & 90 & 0 & 0 \\
\hline BRS 220 & P. Grossa/PR ${ }^{z}$ & I & 24 & 44 & 12 & 0 \\
\hline CD 116 & Castro/PR & I & 20 & 36 & 3 & 0 \\
\hline Mirante & P. Grossa/PR & I & 16 & 44 & 16 & 0 \\
\hline CD 114 & Cascavel/PR & II & 0 & 0 & 0 & 100 \\
\hline CD 117 & Cascavel/PR & II & 0 & 0 & 0 & 100 \\
\hline Marfim & Entre Ijuís/RS & II & 4 & 80 & 16 & 0 \\
\hline Campeiro & Entre Ijuís/RS & II & 4 & 68 & 16 & 0 \\
\hline Quartzo & Entre Ijuís/RS & II & 14 & 85 & 4 & 0 \\
\hline Mirante & Entre Ijuís/RS & II & 10 & 80 & 0 & 0 \\
\hline Horizonte & Santa Rosa/RS & II & 0 & 74 & 3 & 0 \\
\hline Safira & Santa Rosa/RS & II & 0 & 96 & 0 & 0 \\
\hline Mirante & Santa Rosa/RS & II & 0 & 63 & 0 & 0 \\
\hline Quartzo & Ventania/PR & II & 0 & 74 & 0 & 0 \\
\hline Horizonte & Santo Augusto/RS & II & 0 & 72 & 0 & 0 \\
\hline Mirante & Santo Augusto/RS & II & 6 & 93 & 3 & 0 \\
\hline Itaipu & Santo Augusto/RS & II & 0 & 75 & 3 & 0 \\
\hline Quartzo & Santo Augusto/RS & II & 0 & 71 & 0 & 0 \\
\hline Guamirim & Santo Augusto/RS & II & 12 & 80 & 4 & 0 \\
\hline Pioneiro & Santo Augusto/RS & II & 20 & 64 & 0 & 0 \\
\hline BRS Tang. ${ }^{z}$ & Tibagi/PR & II & 8 & 40 & 0 & 0 \\
\hline CD 116 & Tibagi/PR & II & 12 & 32 & 0 & 0 \\
\hline BRS 220 & Tibagi/PR & II & 35 & 30 & 20 & 0 \\
\hline BRS Tang. & Itapeva/SP & II & 4 & 61 & 0 & 0 \\
\hline Pardela & Arapongas/PR & III & 4 & 92 & 0 & 0 \\
\hline CD 116 & Arapongas/PR & III & 0 & 90 & 0 & 0 \\
\hline ONIX & Indianópolis/MG & IV & 0 & 72 & 0 & 0 \\
\hline BRS 264 & Indianópolis/MG & IV & 16 & 68 & 0 & 0 \\
\hline BRS 254 & Indianópolis/MG & IV & 12 & 64 & 0 & 0 \\
\hline Média & & & 7,5 & 66,3 & 3,1 & 6,2 \\
\hline Frequência & & & 55,9 & 100,0 & 38,2 & 8,8 \\
\hline
\end{tabular}

Na análise das amostras da safra 2010, o fungo D. tritici-repentis também apresentou maior frequência e incidência sendo que apenas os fungos dos gêneros Drechslera e Bipolaris foram detectados (Tabela 3). A partir da safra 2006, tem sido observado dificuldade de controle da mancha-amarela em diversas regiões do Brasil. Sendo D. triticirepentis o fungo com maior incidência e frequência, é provável que tenha ocorrido redução de sua sensibilidade aos fungicidas empregados nas lavouras de trigo.

Dados referentes à safra 2011, mostram a ocorrência dos fungos $D$. tritici-repentis, $B$. sorokiniana, D. siccans e $S$. nodorum, sendo que a presença de $S$. nodorum foi registrada somente nesta safra, não se observando nas demais (Tabela 4).
Nota-se que, em nenhuma das amostras analisadas (estados do Paraná, Rio Grande do Sul, Minas Gerais, São Paulo e Santa Catarina) foi identificado o fungo $S$. tritici. Conforme Reunião (15), as principais manchas foliares do trigo, na região sul do Brasil, são a mancha-amarela da folha, causada por Drechslera spp., a mancha marrom causada por B. sorokiniana e a septoriose causada por $S$. nodorum; os resultados encontrados no presente estudo confirmam o citado em Reunião (15).

Considerando as 162 amostras analisadas (Tabela 5), na média, B. sorokiniana apresentou incidência de $7,6 \%$ e frequência de $53,1 \%, D$. tritici-repentis com incidência de 59,2 $\%$ e frequência de $90,6 \%$, D. siccans apresentou incidência e frequência 
Tabela 5. Detecção de fungos em manchas foliares de trigo durante as safras agrícolas de 2008 a 2011. Passo Fundo/RS, 2012

\begin{tabular}{|c|c|c|c|c|c|c|c|c|c|}
\hline \multirow[t]{2}{*}{ Safra } & \multicolumn{2}{|c|}{ Bipolaris sorokiniana } & \multicolumn{2}{|c|}{ Drechslera tritici-repentis } & \multicolumn{2}{|c|}{ Drechslera siccans } & \multicolumn{2}{|c|}{ Stagonospora nodorum } & \multirow[t]{2}{*}{ № de amostras } \\
\hline & $I^{x}(\%)$ & $F^{y}(\%)$ & I (\%) & F (\%) & I (\%) & F (\%) & I (\%) & F $(\%)$ & \\
\hline 2008 & 5,9 & 56,2 & 18,0 & 62,5 & 36,0 & 87,0 & $0,0 *$ & 0,0 & 16 \\
\hline 2010 & 2,2 & 30,5 & 86,4 & 100,0 & 2,1 & 33,3 & 0,0 & 0,0 & 36 \\
\hline 2011 & 7,5 & 55,9 & 66,3 & 100,0 & 3,1 & 38,2 & 6,2 & 8,8 & 34 \\
\hline
\end{tabular}

Outros fungos: Fusarium graminearum, Alternaria sp., Epicoccum $\mathrm{sp} .{ }^{*}$ não detectado. ${ }^{\mathrm{x}}$ Incidência, ${ }^{\mathrm{y}}$ Frequência

Tabela 6. Incidência ${ }^{x}$ de fungos associados a manchas foliares em cultivares de trigo na região VCU I, II, III e IV durante as safras agrícolas de 2008 a 2011. Passo Fundo/RS, 2012

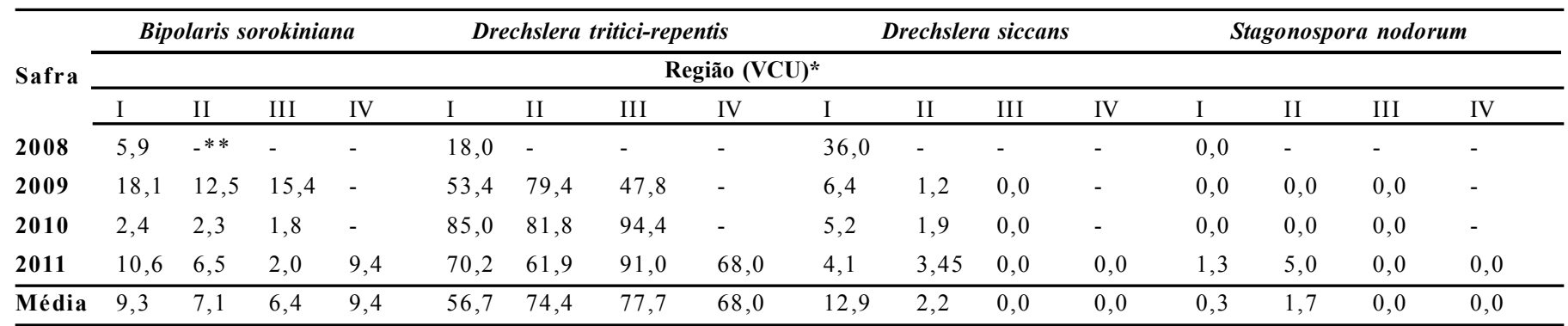

Incidência (\%) determinada em discos de $9,0 \mathrm{~mm}$ de diâmetro recortados de manchas foliares e incubados 25 por amostra. $*$ Valor de Cultivo e Uso.**região não avaliada

de $11,0 \%$ e $48,1 \%$, respectivamente, e $S$. nodorum com incidência de $1,6 \%$ e frequência de $2,2 \%$. Nota-se que nas safras de 2009,2010 e 2011 a maior incidência e frequência foi de $D$. tritici-repentis.

Danelli et al. (7), em estudos de etiologia e intensidade de manchas foliares em cultivares de trigo, em três locais do Rio Grande do Sul/Brasil, relataram que os principais fungos causadores de manchas foliares em Passo Fundo, Santo Augusto e Vacaria foram B. sorokiniana $(0,67 \%)$, D. siccans $(18,0 \%)$, D. tritici-repentis $(43,0 \%)$ e $S$. nodorum $(51,0 \%)$, registrando também a presença de $D$. siccans e o ressurgimento de $S$. nodorum, que conforme os resultados obtidos no trabalho, foi detectada somente na safra 2011.

Prestes et al. (9), em estudos sobre a incidência de manchas foliares em trigo, descreveram que dentre os fungos associados às manchas foliares houve a predominância na seguinte ordem, $S$. nodorum, D. tritici-repentis e B. sorokiniana.

$\mathrm{Na}$ comparação das médias de incidência das regiões de VCU analisadas (Tabela 6), D. tritici-repentis apresentou a maior incidência nas quatro regiões, seguido de $B$. sorokiniana, $D$. siccans e $S$. nodorum. Registrou-se a maior incidência de $D$. tritici-repentis na região de VCU III, considerada quente/ moderadamente seca/baixa $(77,7 \%)$, seguido da região VCU II $(74,4 \%)$, VCU IV (68,0 \%) e região de VCU I (56,7 \%). B. sorokiniana, apresentou maior incidência na região VCU IV. A incidência média nas regiões de VCU I, II, III e IV foi de $9,3 \%$, $7,1 \%, 6,4 \%$ e $9,4 \%$, respectivamente. Por outro lado, para $D$. siccans foi registrado maior incidência na região de VCU I (12,9 \%) e $S$. nodorum com maior incidência na região de VCU II, não sendo detectado sua presença na região de VCU III e IV.

Os dados obtidos mostram que a ocorrência dos fungos associados às manchas foliares em trigo variam conforme $\mathrm{o}$ ano $\mathrm{e} o$ local de cultivo e/ou das condições climáticas predominantes na região, que desempenham importante papel na epidemiologia das doenças.

\section{REFERÊNCIAS BIBLIOGRÁFICAS}

1. Arruda, M.A.; Bueno, C.R.N.C.; Zamprogno, K.C.; Lavorenti, N.A.; Urashima, A.S. Reação do trigo à Magnaporthe grisea nos diferentes estádios de desenvolvimento. Fitopatologia Brasileira, Brasília, v. 30, p. 121-126, 2005.

2. Bohatchuk, D.A.; Casa, R.T.; Bogo, A.; Kuhnem, P.J.; Reis, E. M.; Moreira, E.N. Modelo de ponto crítico para estimar danos de doenças foliares do trigo em patossistema múltiplo. Tropical Plant Pathology, Brasília, v. 33, n. 5, p. 363-369, 2008.

3. Casa, R.T.; Reis, E.M.; Bezerra, R.; Herok, P.; Silva, A. Efeito de manchas foliares no rendimento de grãos de trigo. Fitopatologia Brasileira, Brasília, v. 26, p. 445, 2001.

4. Casa, R.T.; Reis, E.M.; Blum, M.M.C. Critério: Limiar de Dano Econômico (LDE) e quantificação de danos. Em: Reis, E.M. (Org.). Critérios indicadores do momento para aplicação de fungicidas visando ao controle de doenças em soja e trigo. Passo Fundo: Ed. Aldeia Norte, 2009. 148 p.

5. CONAB (Companhia Nacional de Abastecimento). Acompanhamento de safra brasileira: último levantamento, 2012. Disponível em: <http://www.conab.gov.br>. Acesso em: 14 jan. 2012.

6. Cunha, G.R.; Scheeren, P.L.; Pires, J.L.F.; Maluf, J.R.T.; Pasinato, A.; Caierão, E.; Silva, M.S.; Dotto, S.R.; Campos, L.A.C.; Felício, J.C.; Castro, R.L.; Marchioro, V.; Riede, C.R.; Rosa Filho, O.; Tonon, V.D.; Svoboda, L.H. Regiões de adaptação para trigo no Brasil. Passo Fundo/Embrapa Trigo, 2006. 35 p. (Circular Técnica Online, 20).

7. Danelli, A.L.D.; Reis, E.M.; Fiallos, F.R.G. Etiologia e intensidade de manchas foliares em cultivares de trigo em três locais do Rio Grande do Sul, Brasil. Scientia Agropecuaria. v. 2, p. 149-155, 2011.

8. Metha, Y.R. Manejo integrado de enfermedades del trigo. Imprenta Landivar, Santa Cruz de la Sierra: Bolívia. 1993. 319 p.

9. Prestes, A.M.; Santos, H.P. dos; Reis, E.M. Práticas culturais e incidência de manchas foliares em trigo. Pesquisa Agropecuária Brasileira, Brasília, v. 37, n. 6, p. 791-797, 2002.

10. Rees, R.G.; Platz, G.J. Effects of yellow spot on wheat: Comparision of epidemics at different stages of crop development. Australian Journal of Agricultural Research Victoria, n. 34, p. 39-46, 1983. 
11. Reis, E.M.; Casa, R.T.; Medeiros, C.A. Diagnose, patometria e controle de doenças de cereais de inverno. Londrina: E. S. Comunicação, 2001. 94 p.

12. Reis, E.M.; Medeiros, C.A.; Casa, R.T. Control of leaf blights of wheat by elimination of inoculum source. In: Duveiller, E.; Dubin, H.J.; Reeves, J.; McNab, A. (Org.) Helminthosporium blights of wheat: spot bloch and tan spot. CIMMYT, El Batan: Mexico.1998. p. 327-332.

13. Reunião da Comissão Sul-Brasileira de Pesquisa de Trigo, 37., 2005 Cruz Alta. Indicações técnicas da Comissão Sul-Brasileira de Pesquisa de Trigo: trigo e triticale. Cruz Alta: FUNDACEP, 2005. $162 \mathrm{p}$.
14. Reunião da Comissão Sul-Brasileira de Pesquisa de Trigo, 38., 2006 , Passo Fundo. Indicações técnicas para a cultura do trigo. Passo Fundo: Embrapa Trigo, 2006. 114 p.

15. Reunião da Comissão Brasileira de Pesquisa de Trigo e Triticale. Informações técnicas para trigo e triticale: safra 2012. Dourados: Embrapa Agropecuária Oeste, 2011. 204 p.

16. Stolte, R.E. Sensibilidade de Bipolaris sorokiniana e de Drechslera tritici-repentis a fungicidas in vitro. 2006. 92 p. Dissertação (Mestrado em Agronomia) - Universidade de Passo Fundo.

17. Zambolim, L.; Casa, R.T.; Reis, E.M. Sistema plantio direto e doenças em plantas. Fitopatologia Brasileira, Brasília, v. 25, p. $585-$ $595,2000$. 